\title{
A Dual-Band Conformal Antenna for GNSS Applications in Small Cylindrical Structures
}

\author{
Kazeem A. Yinusa, Member, IEEE
}

\begin{abstract}
A non-planar circularly polarized dual-band antenna for global navigation satellite system is presented. The shape of the antenna is conformal to the surface of a cylinder such that the antenna can be completely integrated within the body of a cylindrical carrier without protruding components. In order to enable multi-constellation satellite navigation, the antenna is resonant at the Galileo E5a/b and E1 including GPS and Glonass bands. The far-field patterns of the simulated and manufactured antenna show a wide angular coverage over the upper hemisphere. The antenna design concept would enable the integration of antenna arrays on safety critical small aeronautical platforms where array signal processing with structurally integrated antennas are preferred due to drag and other safety considerations.
\end{abstract}

Index Terms-Conformal Antenna, Structurally Integrated Antenna, Satellite Navigation, GPS.

\section{INTRODUCTION}

$\mathbf{G}$ AIN and axial ratio are well-known metrics with which the performance of antennas used in global navigation satellite systems (GNSS) are evaluated. Both performance metrics measure the ability of the antenna to efficiently receive the very weak satellite signals as they arrive at the receiver and the antenna's ability to suppress multipath signals which distort the computed pseudoranges of the receiver from the satellites. In order to improve the availability of the navigation system and to reduce the geometric dilution of precision, it is important that the performance of the antenna does not degrade considerably at low elevations. Good antenna performance at low elevation angles allows a GNSS receiver to reduce its elevation mask as signals are received with acceptable signal-to-noise ratio from more satellites. This requirement has motivated research and development of antennas with wide angular coverage in terms of gain and axial ratio [1]-[3].

As GNSS finds new applications including those that are safety critical, new concerns have emerged such as the threat of intentional and non-intentional interference. Non-intentional interference can arise, for instance, due to active personal privacy devices (PPDs) or jammers in the vicinity of the receiver. Intentional interference is less frequent but it is increasingly becoming important. It can arise from jamming devices installed at certain locations in order to disrupt navigation services, e.g. against unmanned aerial vehicles (UAVs) for security or privacy reasons. More sophisticated category of intentional interference are due to spoofers who transmit GNSS-like signals in order to gain control over the target's navigation system.

The authors is with the institute for communications and navigation at the German Aerospace Center, 82234 Wessling, Germany (e-mail: kazeem.yinusa@dlr.de).
Researchers have developed several solutions for safety critical satellite navigation for multipath suppression, interference mitigation, protection against spoofers, enhancement of signal availability and to improve the precision of the obtained receiver position [4]-[12]. An important aspect in most of these solutions is an antenna array because of the additional degree of freedom that they provide. In [10], for instance, an antenna array is utilized to estimate the direction of arrival of the jamming signal and a beamforming algorithm is used to place a null towards the jammer and steer the beam of the antenna towards the satellite. In most applications, however, only a limited space is available for the integration of the antenna. The benefits of antenna arrays coupled with the limited space has led to the development of miniaturization techniques for GNSS antennas and methods to reduce the accompanying mutual coupling [13], [14]. However, planar arrays still have some drawbacks when integrated on small non-planar aeronautical structures e.g. on UAVs. Firstly, planar antenna arrays create structural discontinuities when they are integrated on non-planar surfaces and this can be undesirable since it increases drag and may degrade the aesthetics of the structure. Secondly, the coverage angle of planar arrays degrade at low elevation where good antenna performance are often desirable e.g. to suppress multipath signals. These drawbacks have led to the development of antenna arrays that conform to the structure where they are integrated [15], [16]. These antennas take advantage of the shape of the carrier which can be naturally suited for omnidirectional coverage e.g. spherical or cylindrical shapes.

In this letter, a dual-band patch antenna resonant at the Galileo E5a/b and E1 including GPS and Glonass bands is presented. The antenna is conformal to the surface of a cylinder such that it can be completely integrated within the body of a cylindrical carrier without protruding components. In order to obtain circular polarization over a wide angular range, the antenna is fed by four feeds with appropriate phase shifts. In [17], an analysis of a cylindrical-circular patch antenna was presented to understand the effects of curvature on the $\mathrm{TM}_{11}$ mode. The proposed antenna in this paper utilizes such a cylindrical-circular patch configuration to obtain a flushmounted circularly polarized antenna on an electrically small cylinder. The antenna utilizes four azimuthally symmetric distributed probes in order to suppress the higher order modes which become more resonant with smaller cylinder radius. The installed performance of the antenna is also demonstrated by simulation on a small UAV. The shape of the antenna will allow a systematic arrangement of the antenna into a conformal array on small cylindrical platforms. 


\section{ANTENNA DESIGN}

The planar layout of the antenna is shown in Fig. 1. It consists of two stacked circular patches on a flexible dielectric substrate. The lower patch radiates at the E5 band with central frequency of $1.189 \mathrm{GHz}$ while the upper patch radiates at the E1 frequency band with central frequency of $1.575 \mathrm{GHz}$. The vertical and horizontal current modes of the patch can

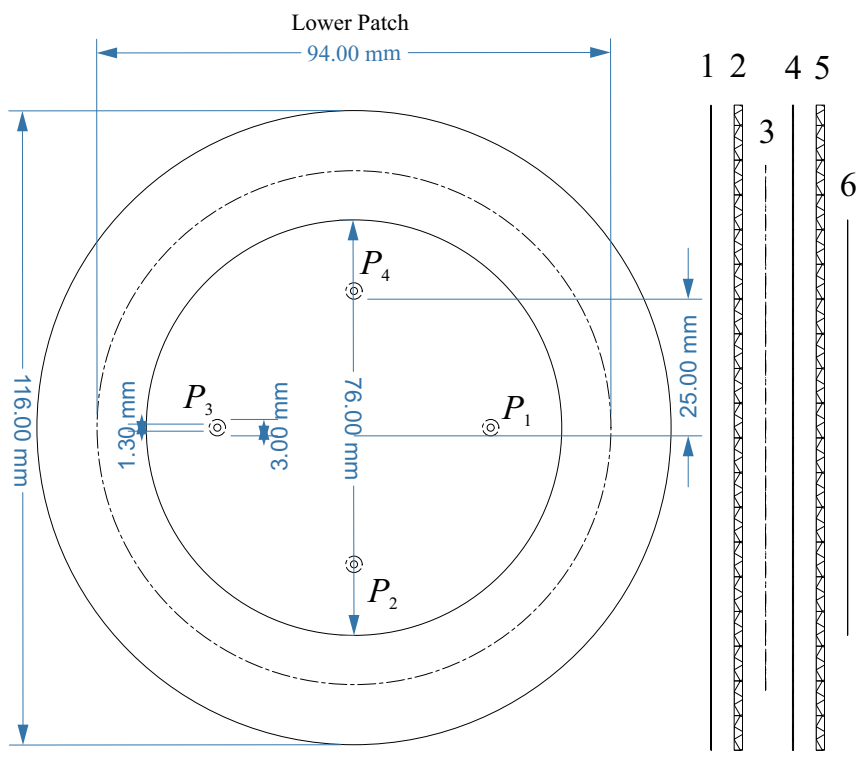

Fig. 1. Layout and stack-up of the planar antenna without wrapping. Layers 1,3 and 6 are copper layers with a thickness of $17 \mu \mathrm{m}$ while layers 2 and 5 are Rogers Isoclad ${ }^{\mathrm{TM}} 933$ layers with relative permittivity of 2.33 and a thickness of $1.524 \mathrm{~mm}$. Layer 4 is the $3 \mathrm{M}^{\mathrm{TM}}$ transfer tape 465 with a thickness of $0.05 \mathrm{~mm}$.

be excited at feed points $p_{1}$ and $p_{2}$, respectively. Circular polarization is then obtained in the boresight direction by combining these modes with the same amplitude and a phase difference of $90^{\circ}$. For a planar circular patch, an arbitrary feed point is usually chosen at an optimized radius while the location of the second feed is chosen such that the two feeds are at an angle of $90^{\circ}$. When the circular patch is imposed on a cylinder to obtain a conformal shape as in Fig. 2, the resonant frequency of the two orthogonal modes are affected to a different extent by the bend with the mode along the curvature of the cylinder experiencing a larger reduction in the resonant frequency as shown in the result of the eigen mode solution in the left axis of Fig. 3. A frequency shift of up to $1.5 \%$ is observed for the mode along the cylinder curvature which is significant considering the typically narrow bandwidth of patch antennas. The difference in the resonance frequencies of the first two modes results in a deteriorating axial ratio in the boresight direction as the antenna is wrapped on smaller cylinders [18].

\section{A. Effects of higher order modes}

The neighboring modes to the two boresight modes $\left(\mathrm{TM}_{11}\right)$ on a planar circular patch are the lateral modes such as $\mathrm{TM}_{(2,3,4) 1}$. These modes radiate mainly in the middle to lower elevations and have also been exploited for circular polarization within these angular ranges [19]. For a planar

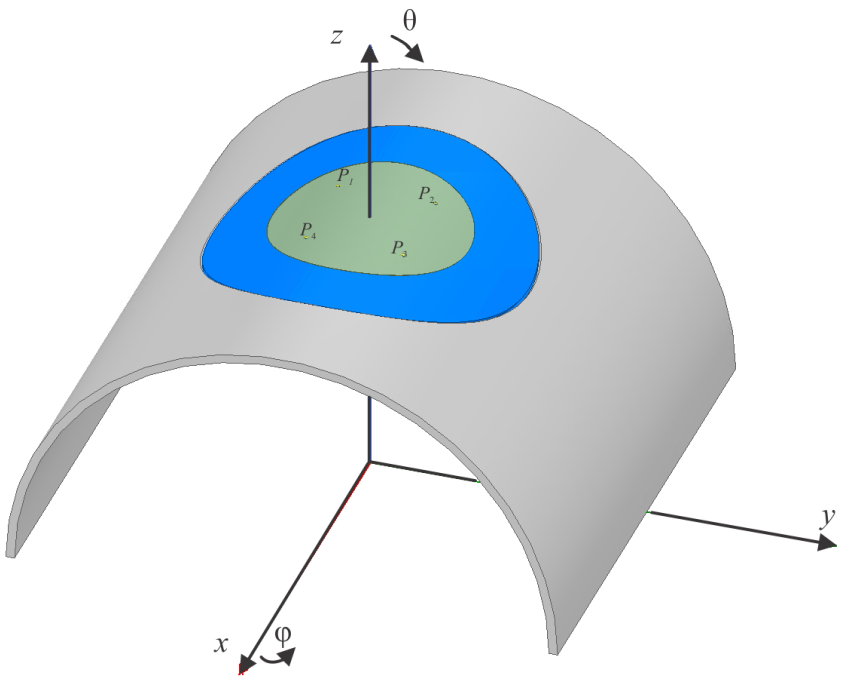

Fig. 2. The conformal antenna model flush-mounted in a cylindrical metal surface with a radius of $10 \mathrm{~cm}$.

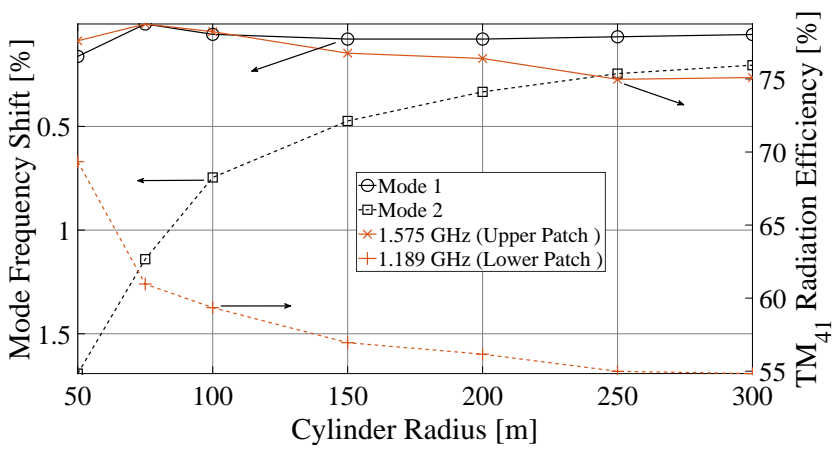

Fig. 3. The two main effects of curvature on the patch antenna. Left axis: obtained frequency shift (compared to a planar circular patch) in the horizontal and vertical modes of the single (lower) layer patch antenna from the layout in Fig. 1 when it is wrapped on a cylinder with variable radius. Mode 1 is along the curvature of the cylinder while mode 2 is along the axis of cylinder. Right axis: The increasing radiation efficiency of the unwanted $\mathrm{TM}_{41}$ mode with strong cylinder curvature.

circular patch antenna, the contribution of these higher order modes are relatively small at the resonance frequency of the desired $\left(\mathrm{TM}_{11}\right)$ mode and are usually ignored when designing planar antennas. However, when the patch is wrapped on a cylinder, the contributions of these modes begin to increase with a decrease in the radius of the cylinder. This can be observed in the right axis of Fig. 3 which shows the radiation efficiency of the higher order mode $\mathrm{TM}_{41}$ against the cylinder radius. The resonance behavior of this mode was simulated by exciting the antenna in Fig. 2 with a phase shift of $0^{\circ}, 90^{\circ}$, $0^{\circ}, 90^{\circ}$ at ports $p_{1}, p_{2}, p_{3}$ and $p_{4}$, respectively.

\section{B. Antenna parameters and far-field patterns}

The single element consists of two stacked patches on a dielectric substrate flush-mounted on a cylinder with a radius of $100 \mathrm{~mm}$. The planar antenna with its dimensions are shown in Fig. 1 and the manufactured antenna is shown in Fig. 4. The layers are manufactured separately and are binded together 
by a transfer tape and aluminum screws. In order to remove the contributions of the higher order modes, the antenna is additionally fed at two locations $\left(p_{3}\right.$ and $p_{4}$ in Fig. 2). A phase shift of $0^{\circ}, 90^{\circ}, 180^{\circ}$ and $270^{\circ}$ is applied to the feeds, respectively, at $p_{1}, p_{2}, p_{3}$ and $p_{4}$ to suppress the higher order modes. The required phase shifts at the feeds are obtained by means of a 1 to 4 wideband commercial off-the-shelf power divider/combiner. This way, the circular polarization purity of the antenna is less affected when it is conformally mounted on a cylindrical platform with strong curvature. The manufactured antenna was measured in a near-field measurement chamber equipped with a spherical positioner in order to obtain its farfield pattern. The obtained frequency response of the antenna gain at zenith can be seen in Fig. 5 where the resonance of the antenna at the required frequency bands can be observed. The realized gain pattern and axial ratio of the antenna are shown in Fig. 6 and Fig. 7 for E5 central frequency of $1.189 \mathrm{GHz}$ and E1 central frequency of $1.575 \mathrm{GHz}$, respectively. In Fig. 8, the performance of the proposed antenna is compared to a conventional two-feed antenna where the feeds are located along the $x$ - and $y$-axis. It can be observed that the presented antenna configuration produces lower cross polarization levels and the gain patterns are more symmetric around zenith compared to the antenna with two feeds.

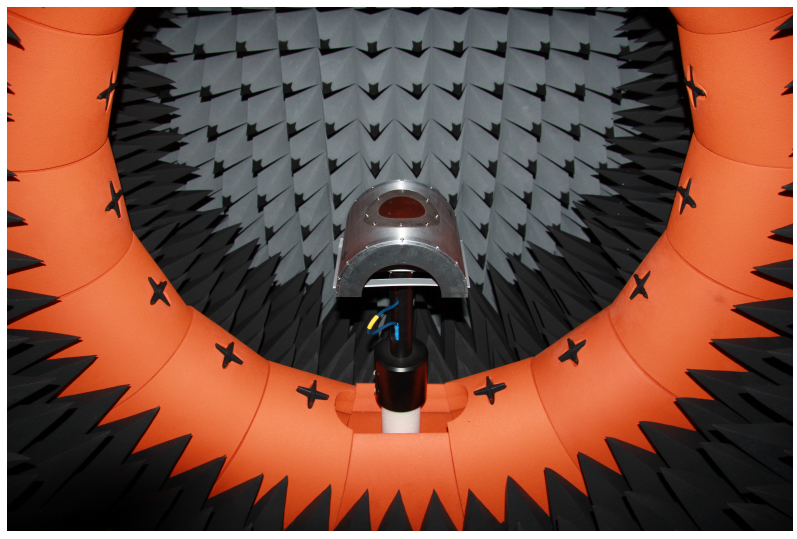

Fig. 4. The manufactured conformal antenna in a measurement chamber.

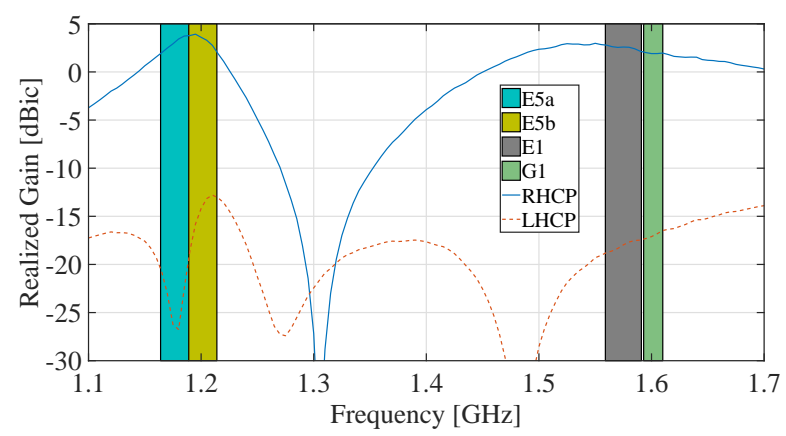

Fig. 5. Measured realized gain at zenith shows the resonance of the proposed antenna at the E5a/b and E1 frequency bands.
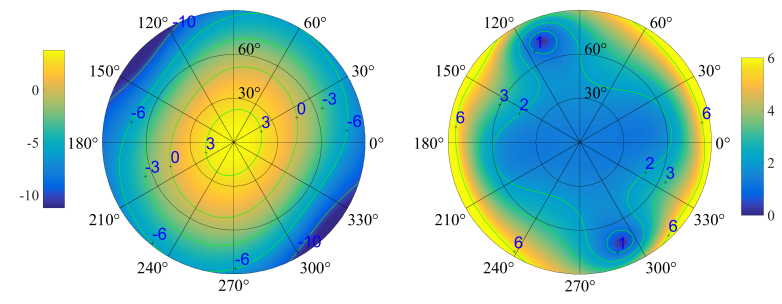

Fig. 6. Measured RHCP realized gain [dBic] (left plot) and axial ratio [dB] (right plot) of the antenna at E5 central frequency of $1.189 \mathrm{GHz}$
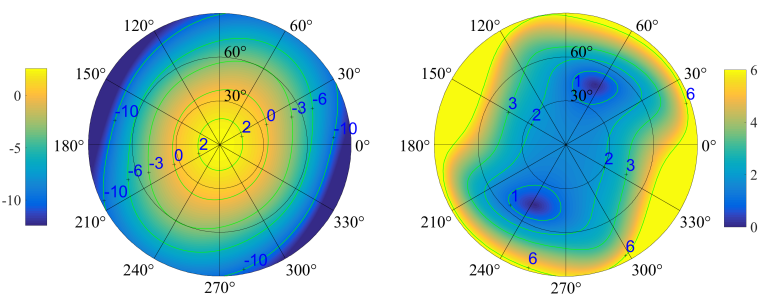

Fig. 7. Measured RHCP realized gain [dBic] (left plot) and axial ratio [dB] (right plot) of the antenna at E1 central frequency of $1.575 \mathrm{GHz}$

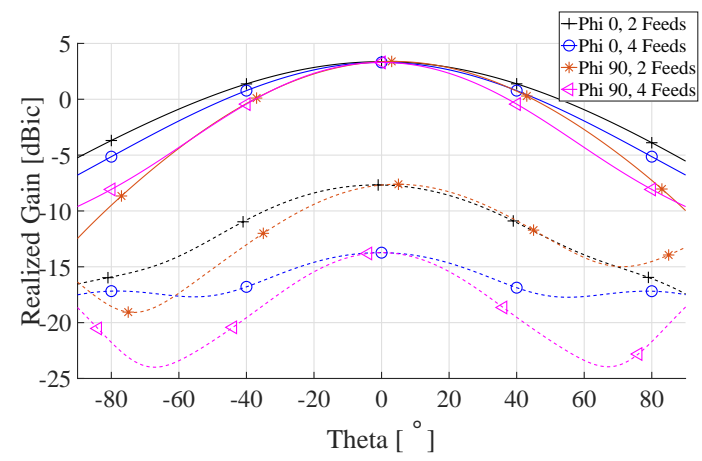

(a) Frequency $=1.189 \mathrm{GHz}$

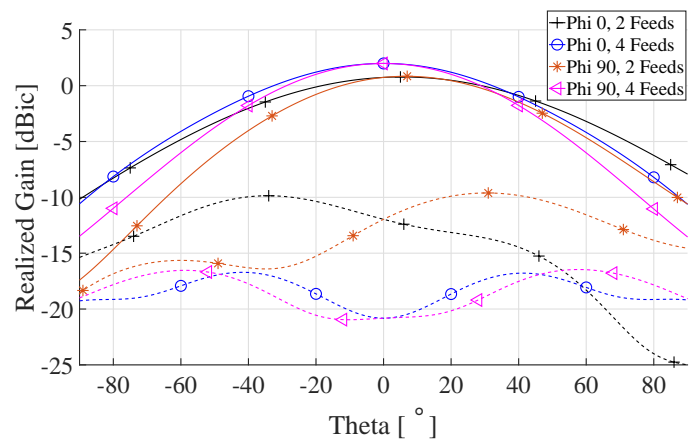

(b) Frequency $=1.575 \mathrm{GHz}$

Fig. 8. Simulated RHCP (continuous lines) and LHCP (dotted lines) realized gain cuts of the four feeds antenna in Fig. 2 compared to the patterns obtained with the same antenna fed with two feeds located along the $x$ - and $y$-axis.

\section{Installed Performance Simulation}

The installed performance of the designed antenna was simulated by integrating it on a small UAV shown in Fig. 9. The wingspan of the UAV is $3.2 \mathrm{~m}$ and the radius of the 
fuselage is about $20 \mathrm{~cm}$. The RHCP gain pattern of the simulated installed antenna is shown in Fig. 10. A particular advantage of the antenna shape is that the concern about antenna size and weight is alleviated especially for antenna arrays since the antenna replaces some parts of the platform.

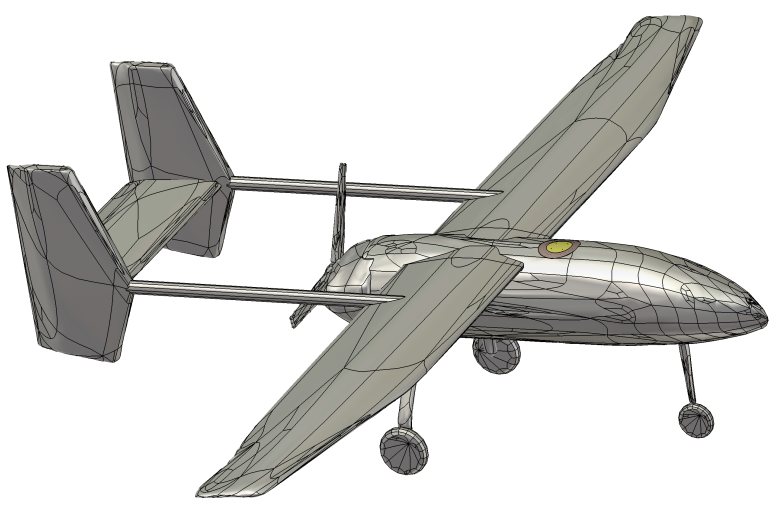

Fig. 9. A 3D model of the conformal antenna integrated on the model of DLR's "Prometheus" UAV.

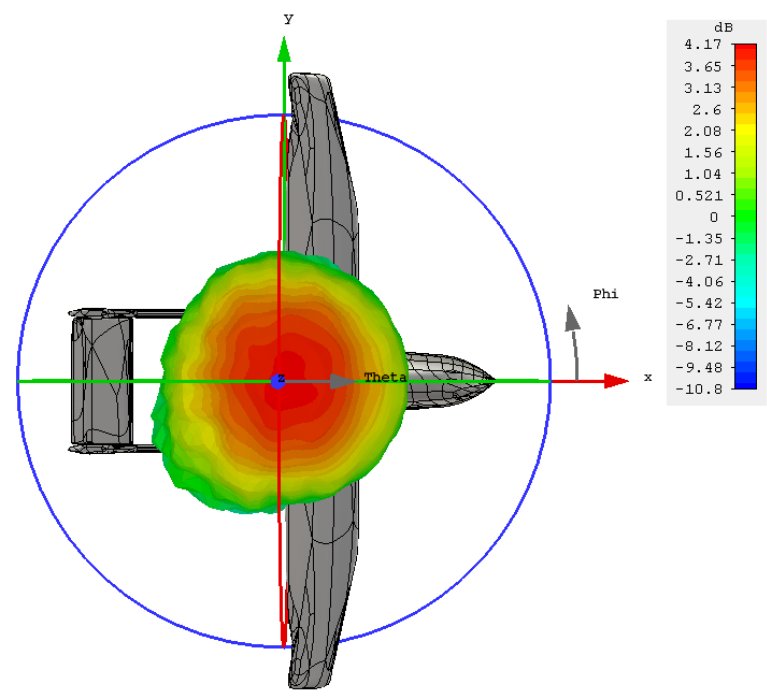

Fig. 10. Simulated RHCP gain pattern of the installed antenna on the UAV at $1.189 \mathrm{GHz}$.

\section{CONCLUSION}

The design and performance of a conformal dual-band patch antenna operating in the L-band has been presented. The main objective of the design is to obtain circular polarization over a wide angular range with an antenna that is conformally flushmounted in a small cylindrical platform without protruding components. In order to suppress higher order modes the antenna is fed with two additional feeds with appropriate phase shifts. The simulated results show relatively good realized gain and axial ratio performance compared to bent conventional two-feeds patch antennas. The performance of the antenna on a small UAV was also demonstrated by means of an installed performance simulation. The properties of the antenna will be useful for small aeronautic structures to achieve array configuration for reliable navigation without increasing aerodynamic drag.

\section{ACKNOWLEDGMENT}

The author would like to thank the colleagues at DLR's Unmanned Aircraft Department, Institute of Flight Systems, for providing the $3 \mathrm{D}$ model of the UAV.

\section{REFERENCES}

[1] K. M. Mak and K. M. Luk, "A circularly polarized antenna with wide axial ratio beamwidth," IEEE Transactions on Antennas and Propagation, vol. 57, no. 10, pp. 3309-3312, Oct 2009.

[2] L. Chen, T. L. Zhang, C. Wang, and X. W. Shi, "Wideband circularly polarized microstrip antenna with wide beamwidth," IEEE Antennas and Wireless Propagation Letters, vol. 13, pp. 1577-1580, 2014.

[3] Y. Luo, Q. X. Chu, and L. Zhu, "A low-profile wide-beamwidth circularly-polarized antenna via two pairs of parallel dipoles in a square contour," IEEE Transactions on Antennas and Propagation, vol. 63, no. 3, pp. 931-936, March 2015.

[4] S.-S. Hwang and J. J. Shynk, "Multicomponent receiver architectures for gps interference suppression," IEEE Transactions on Aerospace and Electronic Systems, vol. 42, no. 2, pp. 489-502, April 2006.

[5] J. Lambert, C. Balanis, and D. DeCarlo, "Spherical cap adaptive antennas for gps," Antennas and Propagation, IEEE Transactions on, vol. 57, no. 2, pp. 406-413, Feb 2009.

[6] M. V. T. Heckler, M. Cuntz, A. Konovaltsev, L. A. Greda, A. Dreher, and M. Meurer, "Development of robust safety-of-life navigation receivers," IEEE Transactions on Microwave Theory and Techniques, vol. 59, no. 4, pp. 998-1005, April 2011.

[7] C. J. Wullems, "A spoofing detection method for civilian 11 gps and the e1-b galileo safety of life service," IEEE Transactions on Aerospace and Electronic Systems, vol. 48, no. 4, pp. 2849-2864, October 2012.

[8] L. Heng, D. B. Work, and G. X. Gao, "Gps signal authentication from cooperative peers," IEEE Transactions on Intelligent Transportation Systems, vol. 16, no. 4, pp. 1794-1805, Aug 2015.

[9] M. L. Psiaki, T. E. Humphreys, and B. Stauffer, "Attackers can spoof navigation signals without our knowledge. here's how to fight back gps lies," IEEE Spectrum, vol. 53, no. 8, pp. 26-53, August 2016.

[10] M. Cuntz, A. Konovaltsev, and M. Meurer, "Concepts, development, and validation of multiantenna gnss receivers for resilient navigation," Proceedings of the IEEE, vol. 104, no. 6, pp. 1288-1301, June 2016.

[11] K. A. Yinusa, W. Elmarissi, S. Caizzone, and A. Dreher, "A multiband conformal antenna array for gnss applications," in 2016 IEEE International Symposium on Antennas and Propagation (APSURSI), June 2016, pp. 897-898.

[12] K. A. Yinusa, L. A. Greda, and A. Dreher, "A conformal multifrequency antenna array for safety-of-life satellite navigation," European Microwave Week, Nuremberg, Germany, Oct. 2017.

[13] H. Steyskal and J. S. Herd, "Mutual coupling compensation in small array antennas," IEEE Transactions on Antennas and Propagation, vol. 38, no. 12, pp. 1971-1975, Dec 1990.

[14] S. Caizzone, "Miniaturized e5a/e1 antenna array for robust gnss navigation," IEEE Antennas and Wireless Propagation Letters, vol. PP, no. 99, pp. 1-1, 2016

[15] S. Raffaelli, Z. Sipus, and P. S. Kildal, "Analysis and measurements of conformal patch array antennas on multilayer circular cylinder," IEEE Transactions on Antennas and Propagation, vol. 53, no. 3, pp. 11051113, March 2005.

[16] L. Josefsson and P. Persson, Conformal Array Antenna Theory and Design. Piscataway, NJ: IEEE Press, 2006.

[17] K.-M. Luk and K.-F. Lee, "Characteristics of the cylindrical-circular patch antenna," IEEE Transactions on Antennas and Propagation, vol. 38, no. 7, pp. 1119-1123, Jul 1990.

[18] T. Kellomäki, "Analysis of circular polarization of cylindrically bent microstrip antennas," International Journal of Antennas and Propagation, vol. 2012, 2012.

[19] J. Huang, "Microstrip antennas for commercial applications," in $\mathrm{Mi}$ crostrip Antennas: The Analysis and Design of Microstrip Antennas and Arrays, D. M. Pozar and D. H. Schaubert, Eds. New York: IEEE Press, 1995, pp. 371-379. 